\title{
Effects of pore fluid salinity on the collapsibility of low plasticity clay
}

\author{
Omar Al Hattamleh ${ }^{1},{ }^{*}$ and M.N. Akhtar ${ }^{2}$ \\ ${ }^{1}$ Civil Engineering Department, College of Engineering Hashemite University, \\ Zarqa, Jordan P.O. Box 150459, Zarqa 13115, Jordan \\ ${ }^{2}$ Civil Engineering Department, College of Engineering, Fahad Bin Sultan University, Tabuk, \\ Kingdom of Saudi Arabia.
}

\begin{abstract}
This paper presents the results on the influence of pore fluid salinity on the Collapsibility of low plastic clay from Hashemite town, Jordan. Double odometer tests were carried out on natural specimens using pore fluids with different salt concentrations. The results indicate a considerable amount of collapse takes place for the sample with increasing the initial applied pressure on the sample regardless of the initial molding water content. However, the dry side of optimum sample shows larger collapse than that on wet side of optimum sample and vice versa. At low applied initial stresses, the higher the salts percentage in the sample, the larger the collapse occurs. Moreover, the amount of collapse tends to increase with increasing the applied pressure to reach a certain value for a given salts percentage, then the amount of collapse asymptotes to its maximum value or level off with increasing the pressure on the sample.
\end{abstract}

\section{Introduction}

Collapsibility of soils which defined as large reduction of volume in soil deposits upon saturation or wetting. This process may or may not be a result of the application of external applied pressure. The collapsible soils appear in strong and stable dry natural state. Upon loading or wetting huge settlement of soil layer occurs and may yield a catastrophic consequences for structures built on it. Such soils are often termed collapsible or metastable soils (among others; Benatti and Miguel, 2013; Mouria et al. 2014; Shalaby, 2014; Al Hattamleh et al. 2015).

Collapse settlement is observed in unsaturated soils of either manmade fills due to poor compaction or in natural soils deposits. The process tends to modify metastable soils and converts it to stable non-collapsible soil after wetting and saturation. The stability of dry collapse soil is attained primarily due to either the presence of thin film of water under tension (i.e. capillarity) or cementation bridge of fine material or presence of salts between large soil particles.

The capillary tension in unsaturated soils seems to vanish after soil pores saturated (i.e. filled with water). In the case of dry soils, the bond between soil particles of fine

\footnotetext{
${ }^{*}$ Correspoding author: hattam@,hu.edu.jo
} 
materials and salts is washed away during saturation process, causing soils to collapse. Several criteria are available in the literature for evaluation of the prone of soil to collapse (Jennings and Knight, 1975).

The main objective of this paper is focus on the evaluating of the collapsibility of soil due to the change of pore water fluids.

\section{Experimental program}

\subsection{Material}

The material used for the tests was natural soil deposits from Hashemite town, Jordan. The Liquid limit and plastic limit of the soil are $21 \%$ and $15 \%$, respectively while the specific gravity of the Soil is 2.63 . The grain size distribution of the soil sample indicates that it contains $83 \%$ of fine particles. Based on the consistency test of clean, washed, and dry samples along with grain size distribution, the soil is classified as CL-ML according to the Unified Soil Classification System (USCS). Table 1 contains more information regarding the indices properties of the soil.

Table 3.1 Physical properties of hashemite soil.

\begin{tabular}{|c|c|c|}
\hline \multicolumn{2}{|c|}{ Index Properties } & Value (unit) \\
\hline \multicolumn{2}{|l|}{ Specific gravity } & 2.63 \\
\hline \multicolumn{2}{|c|}{ Natural Water Content (\%) } & 5.3 \\
\hline \multicolumn{2}{|l|}{ Liquid limit (\%) } & 21 \\
\hline \multicolumn{2}{|l|}{ Plastic limit (\%) } & 15 \\
\hline \multicolumn{2}{|l|}{ Plasticity index (\%) } & 6 \\
\hline \multirow{4}{*}{$\begin{array}{c}\text { Grain Size } \\
\text { Distribution (\%) }\end{array}$} & Gravel & 2 \\
\hline & Sand & 15 \\
\hline & Silts & 45 \\
\hline & Clay & 38 \\
\hline
\end{tabular}

\subsubsection{Soil preparation for testing}

The soil was pulverized using rubber-tipped pestle until they pass sieve \# 40 and larger particles was discarded. The soil samples were mixed with different concentrations of brine (Salty water solution). The brine was diluted with distilled water to give a concentration of total dissolved solids of $25 \%$ (undiluted brine), $15 \%, 10 \%, 5 \%$ and $0 \%$. Thereafter, soil samples were subjected to a different soil characterization tests. 


\section{Test results and analysis}

Three sets of double - Oedometer soil specimens $(20 \mathrm{~mm}$ in height and $76 \mathrm{~mm}$ in diameter) were prepared for each testing state. Figure 1 indicates the state of compaction, percent of brine, and number of specimen tested. The chosen soil sample states, as depicted in the Figure 1, were selected to allow soil specimen's to alter its structure from flocculated or bookhouse structures to dispersed or turbostratic structures (Lambe, 1961). The collapse potential tests were conducted according to the ASTM D 5333 - 03. Applied stresses, initial and final heights, thereafter, ratios of initial and final voids were determined for each tested specimen. Voids ratios versus applied stresses for the set of pair samples were plotted. The collapse potential for each pair specimens was determined for all tested cases.

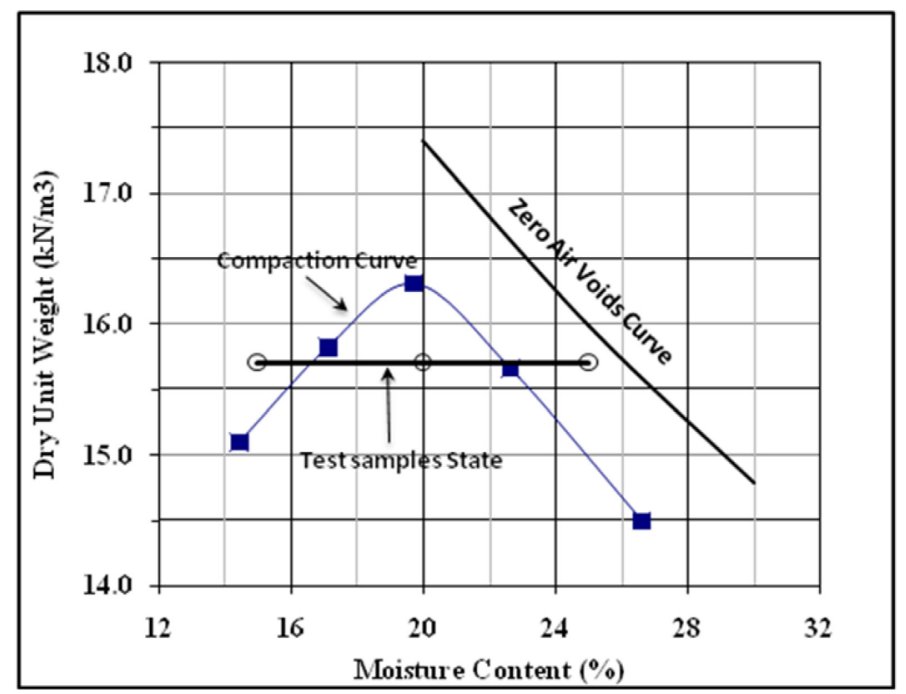

Fig. 1. Compaction curve.

The Oedometer test results for wet and dry, samples of the clay specimens with pore fluid of zero brine at a dry unit weight of $15.7 \mathrm{kN} / \mathrm{m} 3$ and a molding moisture content of 15, 20, and 25 were shown in Figures $2 \mathrm{a}$ to $2 \mathrm{c}$. It is clearly shown from Figures $2 \mathrm{a}$ to $2 \mathrm{c}$ that the soil in both dry and water-inundated samples were hardening with the applying stresses, and a substantial volume decrease occurs. This occurs primarily due to the fact that the soil initial structure was flocculated. With the increasing induced pressure on the samples, the soil structure tries to reach into a new equilibrium structure and tries allied into parallel structure named as disperse structure. 

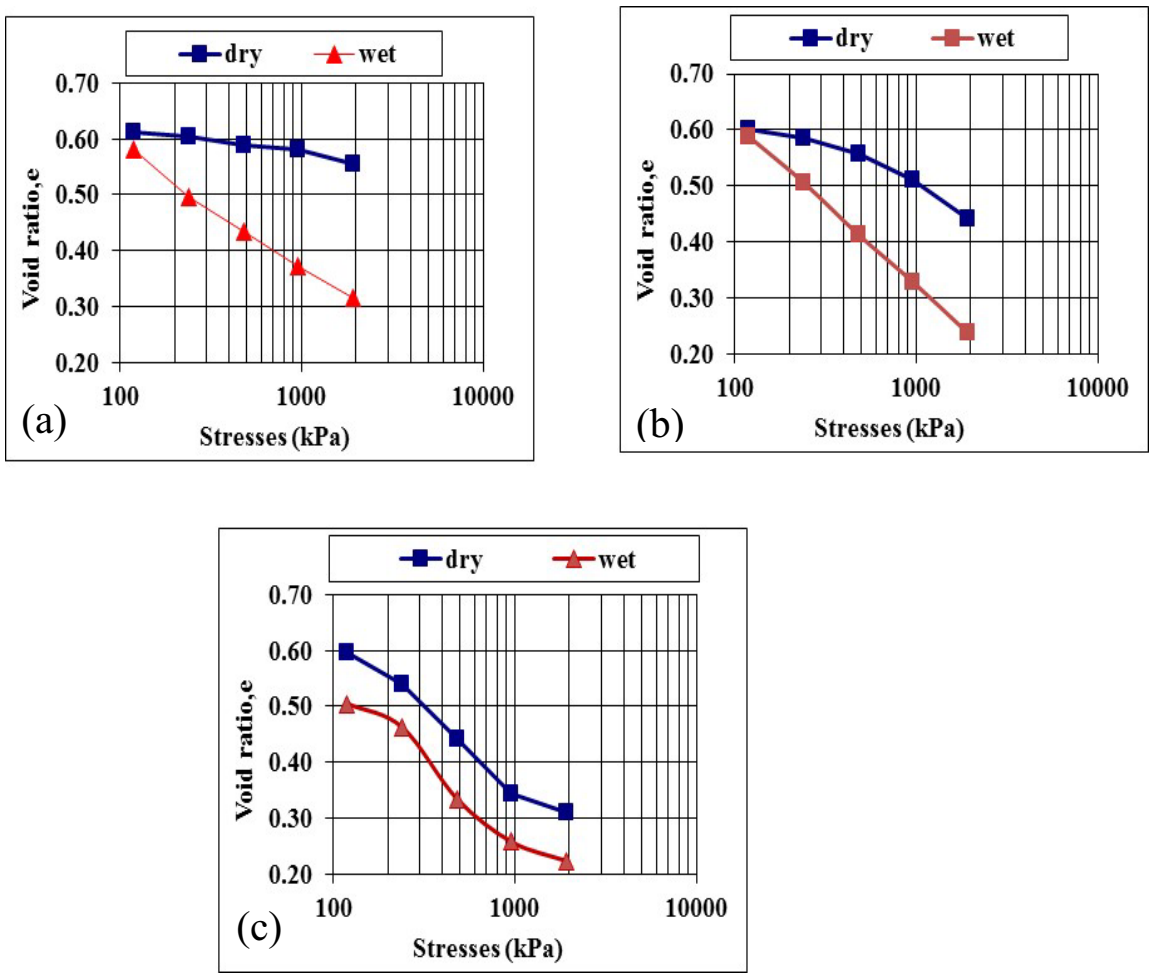

Fig. 2. Compressibility results at dry unit weight of $15.7 \mathrm{kN} / \mathrm{m}^{3}$ and moisture content of (a) $15 \%$ (b) $20 \%$ and (c) $25 \%$.

Figures 3 to 4 show the compressibility curves for the clay sample molded with different percentage of brine fluid. It is obvious from the set of curves depicted in Figures 3 to 4 that the added amount of brine changes the characteristics of collapsibility curve despite the fact that the curves tend to a hardening behavior when the stress applied. Thus the curves typify with those curves of soils with sensitive structure and highly disturbance. The increases of the amount of brine fluid in molding the test specimen obviously increase the concentration of ions. Thus, it will increase the thickness of the diffuse double layer. Therefore, clays structure will favor dispersing structure. 

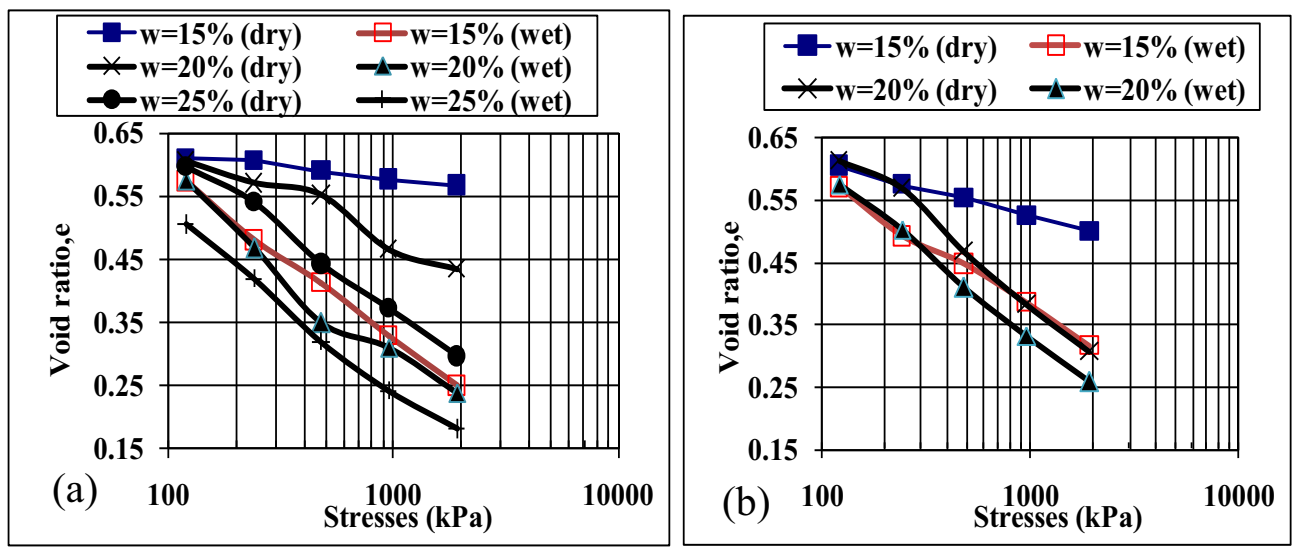

Fig. 3. Compressibility results at dry unit weight of $15.7 \mathrm{kN} / \mathrm{m}^{3}$ and moisture content had shown in the legend and brine content of (a) $5 \%$ (b) $10 \%$.
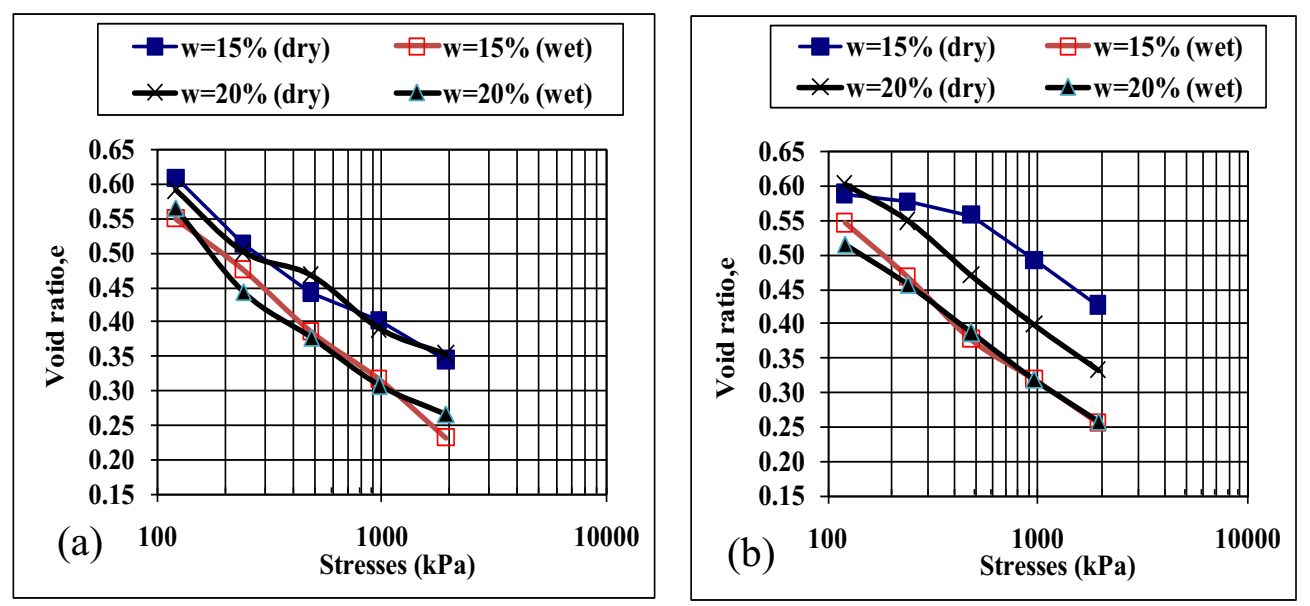

Fig. 4. Compressibility results at dry unit weight of $15.7 \mathrm{kN} / \mathrm{m}^{3}$ and moisture content had shown in the legend and brine content of (a) $15 \%$ (b) $25 \%$.

Collapse potential was evaluated after the dry specimen, while in Oedemeter, reaches the required stress level inundated of brine solution for 24 hours. Thus the collapse potential given as:

$$
C_{p}=\frac{e_{f}-e_{i}}{1+e_{o}}
$$

Where, $\mathrm{e}_{f}=$ final void ratio at the appropriate stress level after wetting, $\mathrm{e}_{i}=$ void ratio at the appropriate stress level before wetting, and $\mathrm{e}_{o}=$ initial void ratio.

Figure 5 shows the variation of collapse potential with applied stresses for different amounts of brine. It is clearly indicated that as the stresses value increases, the amount of collapse increases. Moreover, increasing the amount of salts by weight is not necessary to cause the amount of collapse due to the fact that the salts are not fully washed away when the sample is inundated, which adds an amount of solids to soil solids part, which in this range of applied stress is incrushable. Moreover, the amount of remolding water is deficient to reach an equilibrium amount of water that is fair enough to dissolve all the salt solids. 


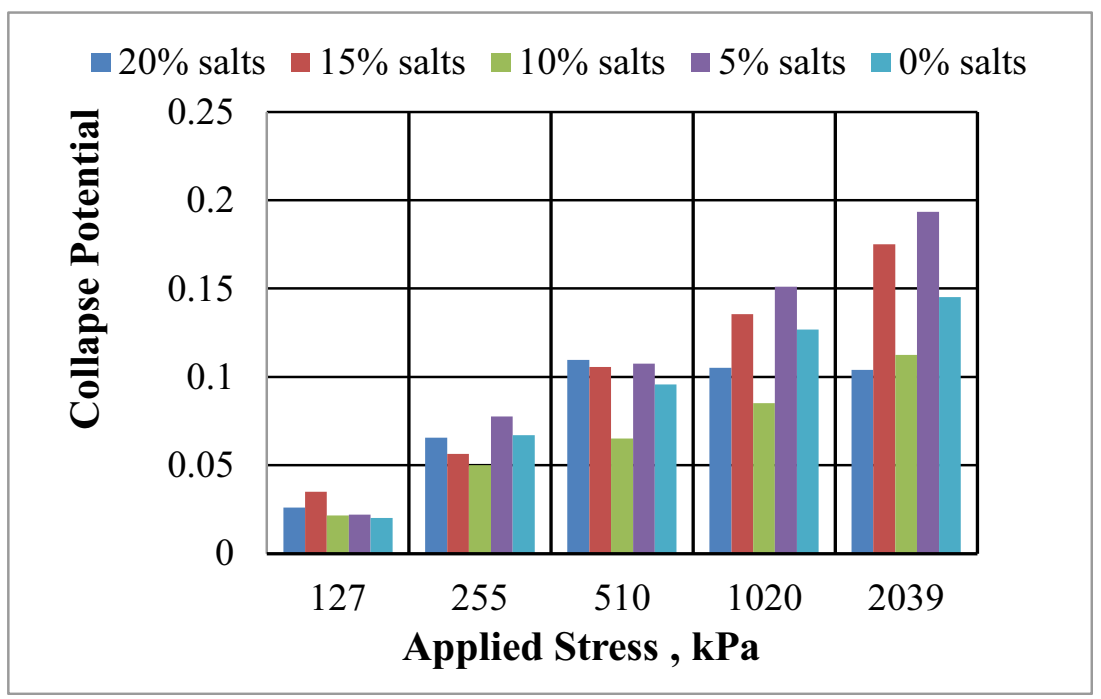

Fig. 5. Variation of collapse potential with stresses for different brine percentage at molding brine content of $20 \%$.

\section{Conclusions}

In the present study, a set of soil samples of low plasticity were prepared and tested to examine the effect of brine in pore fluid in the collapse potential. The brine was mixed thoroughly with soil specimens to the desired concentration. Thereafter, sets of double oedometer tests were conducted on the prepared samples. Based on the results obtained, a considerable amount of collapse takes place for the sample with increasing the induced pressure on the samples despite of the molding water content or brine percentage added. Moreover, samples prepared in molding water content in the dry side of optimum show larger collapse potential more than that on the wet side of optimum samples and vice versa. Furthermore, at low applied induced pressure, the higher the brine percentage added to the samples, the larger the collapse occurs. However, at large induced pressure, the opposite tendency was observed. Lastly, the amount of collapse potential tends to increase with increasing the induced pressure to reach a certain maximum value for a given brine percentage, regardless the additional induced pressure applied on the sample.

\section{References}

1. Benatti J. C. B. and Miguel M. G., A proposal of structural models for colluvial and lateritic soil profile from southwestern Brazil on the basis of their collapsible behaviour. Engineering Geology 153, 1-11 (2013)

2. Mouria G., Shinodab S., Golosovc V., Chalovd S., Shiibae M., Horif T., Okig T., Estimating the collapse of aggregated fine soil structure in a mountainous forested catchment. Journal of Environmental Management 138, 24-31 (2014)

3. Shalaby S.S., The Assessment of the Collapse Potential of Fills during Inundation using Plate Load Tests. Life Science Journal 11, 1001-1006 (2014)

4. Omar H Al Hattamleh, Odeh A. Odeh, Hussein Aldeeky, and M. N. Akhtar, Characterizations of Soil Collapsibility: Effect of Salts Dilution. Journal of Materials and Engineering Structures 2, 33-43 (2015) 
5. Jennings, J.E. and Knight, K., A guide to construction on or with materials exhibiting additional settlement due to collapse of grain structure. Proc. 6th Reg. Conf. Africa on Soil Mechanics and Foundation Engineering 6, 99-105 (1975)

6. Lambe, TW. 1961. Residual pore pressures in compacted clay. Proceeding 5th International Conference on Soil Mechanics and Foundation Engineering. Paris, France.

7. ASTM . Annual Book of ASTM Standards - Section 4: Construction, Volume 04.08 Soil and Rock (1): D420-D5550. American Society for Testing and Materials, USA. 